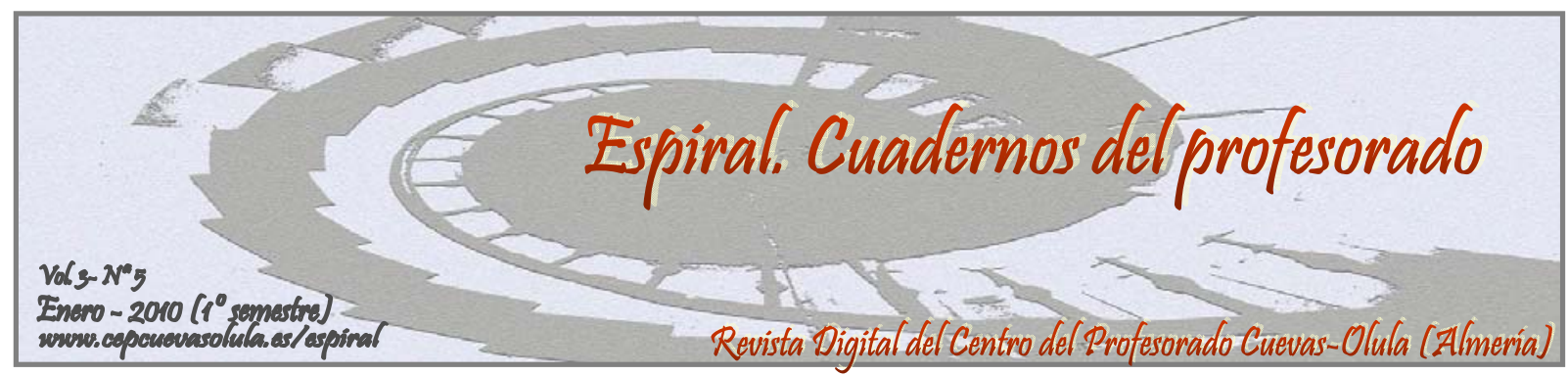

\title{
EL AUXILIAR DE CONVERSACIÓN: UNA FIGURA NECESARIA
}

\section{THE FOREIGN LANGUAGE ASSISTANT: A NECESSARY FIGURE IN THE CLASSROOM}

\section{Cristina Caparrós Cabezas}

\section{Licenciada en Traducción e Interpretación}

RESUMEN: Tanto en colegios de educación primaria como de secundaria, y más concretamente en las clases de idiomas extranjeros, un recurso muy útil y cada vez más demandado es el poder tener un hablante nativo de la lengua extranjera que se está estudiando. El propósito de este trabajo es dar a conocer y describir las distintas medidas que se están llevando a cabo para el fomento de las lenguas extranjeras en nuestro país y explicar de una manera más detallada lo que supone tener a un auxiliar de conversación en el aula. La principal labor de estas personas no es otra que la de ser un mero colaborador lingüístico. No se trata de profesores en sí, básicamente sirven como apoyo al profesorado del departamento de lenguas. Para la elaboración del mismo, tomando como punto de partida la normativa que regula la provisión de estos auxiliares en España, he intentado describir mi propia experiencia personal como auxiliar en el extranjero, así como comparar el sistema educativo británico con el español. Y para terminar, se podría decir que la principal conclusión del presente trabajo es que estas personas no sólo aportan una ayuda extra en las clases de idiomas, sino además la fluidez que una lengua requiere y también experiencias del país al que pertenece, ofreciendo un input cultural tanto para el profesorado como para el alumnado, que beneficia a la hora de introducirse en la cultura meta.

Palabras clave: Idiomas, auxiliar de conversación, beneficios, inmersión, cultura.

ABSTRACT: At Primary or Secondary schools, and mainly at foreign languages lessons, one fantastic resource is having a native speaker of the foreign language that is being studied. The main aim of this work is to know and describe the different measures that the Spanish government is doing to promote foreign languages and explain the role of a foreign language assistant in the classroom. These types of persons are just linguistic collaborators. They are not a full time teachers. Basically, the assistant is useful as a support system for the teachers in the language department. To do this work, I have taken as reference the official law about foreign language assistants and I have tried to describe my own personal experience working as foreign language assistant outside Spain and compare the Spanish and English educational system. As a conclusion, we can say that these people not only provide an extra help in the classroom but also the native-level fluency required and, his or her firsthand experience with the culture of a country in which his or her native language is spoken. Also the assistant provides cultural insights to teachers and students, and also benefits from being immersed in the culture of the host country.

Key words: Foreign languages, language assistant, advantages, immersion, culture.

Caparrós, C. (2010). El auxiliar de conversación: una figura necesaria. Espiral. Cuadernos del Profesorado [en línea], 3(5), 36-43. Disponible en: http://www.cepcuevasolula.es/espiral.

Fecha de recepción: 23/11/2009

Fecha de aceptación: 21/12/2009
Enviar correspondencia a: mcristina.caparros@gmail.com 


\section{1.- INTRODUCCIÓN.}

Los colegios e institutos son un reflejo de la sociedad en la que vivimos, $\mathrm{y}$ al igual que en ésta, no se puede obviar la existencia de personas de diferentes razas, culturas, lenguas y religiones. Todo esto es lo que ha provocado que la sociedad española se haya ganado el término de multiétnica y que se esté trabajando desde diferentes ámbitos (político, cultural, educativo...) para conseguir la interculturalidad, es decir, la convivencia de la heterogeneidad cultural en la que se respetan las minorías, se fomenta la relación entre las culturas y el enriquecimiento mutuo.

Ante esta situación, en los propios colegios, los profesionales de la educación tienen el reto de trabajar en clases multiculturales, para lo que deben estar muy bien preparados, sin embargo, tal y como afirman Encarna Soriano, José Manuel García Argüello y Manuel López, miembros del grupo "Investigación y Evaluación en Educación Intercultural” de la Universidad de Almería $^{1}$, no se puede cargar toda la responsabilidad sobre el profesorado y el centro educativo, y tanto la participación de la familia como de la propia sociedad es fundamental.

Una de las medidas que suponen una ventaja incuestionable para el desarrollo de la interculturalidad, es el conocimiento de idiomas extranjeros ya que la lengua es una vía para comprender otras formas de vida, propagando así valores de tolerancia intercultural.

El idioma no es sólo una manera de referirse a lo que existe en el mundo, sino que conlleva connotaciones compartidas que ayudan al hablante a mantener la sensación de pertenecer a ciertos grupos sociales ${ }^{2}$.

En el actual sistema educativo español y dada la creciente demanda de familias interesadas en que sus hijos e hijas estudien en un segundo idioma, se está dando cada vez más importancia a la introducción de los idiomas extranjeros (generalmente este puesto lo ocupa el inglés) desde edades tempranas y al mismo tiempo, se está apostando por mejorar las competencias lingüísticas y culturales a través de un plan de fomento del plurilingüismo.

1 Para leer el informe completo: http://www.ual.es/GruposInv/EducacionIntercultural/

${ }^{2}$ Michael Byran y Michael Fleming (1998). Perspectivas interculturales en el aprendizaje de idiomas. Press Syndicate of the University of Cambridge
Según un informe realizado por la Unión Europea en 2006 titulado "los europeos y las lenguas”, España ocupa la sexta posición dentro de la Unión Europea, tras países como Hungría, Portugal, Italia, Gran Bretaña e Irlanda, en cuanto al número de personas que no hablan un segundo idioma. Exactamente, en la actualidad, el 56\% de los españoles sólo habla la lengua materna. Sin embargo, en los últimos años, esta actitud parece estar cambiando ya que cada curso aumenta el número de centros que optan por el bilingüismo, así como las medidas y recursos para aprender y fomentar los idiomas extranjeros, ya que se consideran como la puerta de entrada a una Europa cada vez más plurilingüe e integrada.

Andalucía, junto con Madrid, es una de las comunidades españolas más avanzadas y pioneras en cuanto a la introducción y desarrollo de programas bilingües y el fomento de los idiomas. El objetivo es promover el aprendizaje de idiomas en la Comunidad, facilitando las estancias en el extranjero, o tal y como se recoge en el Boletín Oficial de la Junta de Andalucía, "dotar a la población andaluza de las competencias plurilingües y pluriculturales necesarias para hacer frente a los retos que se plantean en nuestra sociedad".

El germen de los programas bilingües andaluces se encuentra entre las páginas del documento "Andalucía: segunda modernización. Estrategias y propuestas para la segunda modernización de Andalucía" (Consejo Asesor para la Segunda Modernización de Andalucía, 2003), creado por un grupo de expertos con el objeto de promover e impulsar cambios que garantizaran la incorporación de Andalucía a la sociedad de la información.

Las recomendaciones de los expertos se resumían en cuarenta propuestas, una de las cuales daría lugar posteriormente al antes mencionado Plan de Fomento del Plurilingüismo ${ }^{4}$, aprobado por la Consejería de Educación de la Junta de Andalucía en 2005 con dos objetivos fundamentales: "Mejorar las competencias plurilingüísticas de la población andaluza en la lengua materna y dotarla, a la vez, de competencias plurilingües y pluriculturales".

3 Para leer el informe completo: http://ec.europa.eu/public_opinion/archives/ebs/ebs_2 43_sum_es.pdf

4

http://www.juntadeandalucia.es/compromisos2008201

2/archivos_repos/0/140.pdf 
Aunque la aprobación del plan data de 2005 (BOJA 5-4-2005), la existencia de programas bilingües en Andalucía se remonta a 1998, cuando la firma de un Protocolo de Colaboración con el Ministerio de Asuntos Exteriores francés, seguido de otro similar con el Cónsul General de Alemania en 2000, permitió a la Consejería de Educación y Ciencia de la Junta de Andalucía la puesta en marcha en cuatro centros escolares, de dos provincias de la comunidad, de secciones bilingües en francés y alemán para la enseñanza de, como mínimo, un área del conocimiento en estas lenguas.

Por lo que se refiere al inglés, la inexistencia de sedes del British Council en Andalucía hizo que hubiera que esperar hasta 2003 para que pudiera firmarse el requerido Protocolo de Colaboración también con este organismo. A partir de entonces, el apoyo del British Council y la celebración del "Año europeo de la ciudadanía a través de la educación" en 2005, dieron un impulso definitivo a los centros bilingües.

El principal objetivo de este trabajo, como ya se ha comentado en la introducción es poder conocer con más exactitud el papel que desarrollan estas personas en el aula de idiomas y describir la experiencia tanto profesional como personal que supuso el poder trabajar como auxiliar de conversación en un país de habla inglesa porque se trata de una figura bastante novedosa que aun no es muy conocida ni por muchos centros educativos ni por los jóvenes españoles que desean y necesitan viajar al extranjero y mejorar sus competencias lingüísticas.

\section{2.- MEDIDAS PARA FOMENTAR LOS IDIOMAS EXTRANJEROS.}

La inminente globalización exige a día de hoy, que los alumnos y alumnas posean mayores destrezas comunicativas en las diferentes lenguas que predominan en el mundo, y particularmente en Europa. Uno de los fenómenos más relevantes en el actual panorama educativo radica en la presencia cada vez más creciente de alumnado procedente de una diversidad étnica, lingüística y cultural. Por eso, como hemos señalado anteriormente, uno de los principales retos de hoy en día no sólo consiste en crear colegios plurilingües sino colegios que sean interculturales.

Para lograr esto, en Andalucía entre algunas otras medidas, se creó el Plan Integral para la
Inmigración de Andalucía en el año 2006 con el que se pretende favorecer que los centros educativos elaboren proyectos que contemplen la perspectiva intercultural, que faciliten $y$ promuevan procesos de intercambio, interacción y cooperación entre las culturas, porque como exponen Cobo et al. (2009), la educación intercultural implica el reconocimiento de la diversidad de culturas como un fenómeno positivo y deseable para todos los centros.

Desde el punto de vista lingüístico y según lo expuesto hasta ahora, es esencial que el alumnado domine en la medida de lo posible más de un idioma ya que así mejoraría la capacidad de comunicación con su entorno.

El inglés es el segundo idioma extranjero estudiado por excelencia en nuestro país y nadie duda de que esta lengua "abre" las puertas laborales a aquellos que lo dominan.

En España, las primeras medidas para fomentar el aprendizaje de idiomas y la adopción del bilingüismo como modelo educativo se remontan, como ya hemos dicho, a 1998. A partir de esta fecha, se han llevado a cabo distintos acuerdos de colaboración y aumentado progresivamente el número de horas de lengua extranjera en los centros.

Otra de las medias que se están llevando a cabo en los centros de enseñanza, y cuyo análisis es el objeto de estas líneas, es la incorporación de la figura de un auxiliar de conversación o asistente de conversación, como también se les conoce. $\mathrm{Y}$ es que la incorporación de estos colaboradores lingüísticos también está reflejada en la ya citado Plan de Fomento del Plurilingüismo.

La Orden de 20 de junio de 2006 por la que se regula la provisión y actividad de los auxiliares de conversación en los centros docentes públicos de la Comunidad Autónoma de Andalucía, contempla la selección de auxiliares de conversación por parte del Ministerio de Educación en virtud de convenios de cooperación bilateral con otros países con la Consejería de Educación de la Junta de Andalucía.

Según un artículo publicado por la Consejería de Educación de la Junta de Andalucía $^{5}$, el pasado curso escolar 2008/2009

\footnotetext{
${ }^{5}$ http://www.juntadeandalucia.es/educacion/nav/conte ni-

do.jsp?pag=/Contenidos/OEE/programasinternacional es/2007_10_17_resolucionAuxiliaresConversacion
} 
hubo 635 auxiliares de conversación en distintos centros escolares de la Comunidad Autónoma seleccionados mediante este procedimiento, de los cuales 75 son financiados por el Ministerio de Educación y Ciencia y 560 por la Consejería de Educación.

Además de esta medida, tanto la Consejería de Educación como las Universidades públicas andaluzas están promoviendo que el alumnado universitario de los últimos años que curse Filología Inglesa, Francesa y Alemana, Magisterio en la especialidad de lenguas extranjeras y Traducción e Interpretación realice prácticas en los Centros Bilingües, y que el alumnado extranjero vinculado a las universidades por medio del programa Erasmus u otros programas internacionales desarrolle actividades en distintos Centros educativos vinculados al Plan de Fomento del Plurilingüismo como colaboradores nativos, o que el alumnado extranjero que realice estudios de postrado en las universidades andaluzas pueda incorporarse a los Centros Bilingües y Escuelas Oficiales de Idiomas en calidad de auxiliares de conversación.

\section{3.- FIGURA DEL AUXILIAR: FUNCIONES.}

El Artículo 2.3 de la Orden de 20 de junio de 2006 es el que regula las distintas funciones que tienen estas personas. Los auxiliares realizan actividades lingüísticas, interculturales, sociolingüísticas y lúdicas, con el objetivo de que los alumnos y alumnas se beneficien de su presencia en la mayor medida de lo posible, y al mismo tiempo potenciar el aprendizaje de los idiomas.

Se trata de colaboradores nativos cuya función en los centros es un importante servicio complementario que apoya la labor del profesorado, posibilitando las prácticas de conversación en lengua extranjera y representando un auténtico vehículo de acercamiento a la cultura de los países donde se habla esa lengua.

Entre las principales tareas que deben llevar a cabo se pueden destacar las siguientes:

- Ayudar al profesorado en las clases de lengua y cultura durante unas 16 horas semanales como hablante nativa y experta sobre temas de su país, con el objetivo de estimular el interés de los alumnos y alumnas en estas áreas.
- Trabajar con grupos reducidos de alumnos para desarrollar habilidades de comunicación oral y escrita y promover el entendimiento cultural.

- Ayudar al profesorado especialista del idioma correspondiente involucrado en el proyecto bilingüe, con la finalidad de mejorar el nivel de los propios docentes y facilitar la elaboración de materiales didácticos para su aplicación en el aula.

De forma más detallada y precisa, las funciones propias de un auxiliar de conversación se encuentran en el artículo 3 de la Orden del 26 de junio de 2006 por la que se regula la provisión y actividad de los auxiliares de conversación en los centros docentes públicos de la Comunidad Autónoma de Andalucía (BOJA 10-7-2006), modificada por la Orden del 14 de septiembre de 2007:

1. Desarrollarán su actividad durante doce horas semanales, que podrá realizarse a tiempo total en un único centro o ser compartida en otro centro adscrito. En cualquier caso, los auxiliares de conversación no serán responsables de la supervisión del alumnado y estarán acompañados siempre en el aula por el profesor o profesora del departamento al que estén apoyando.

2. Posibilitarán la práctica de la conversación oral en la lengua extranjera objeto de estudio del alumnado.

3. Proporcionar un modelo de corrección fonética y gramatical en la lengua extranjera correspondiente.

4. Colaboración con el profesorado en la elaboración de materiales didácticos en la lengua extranjera correspondiente.

5. Acercar al alumnado y al profesorado a la cultura del país donde se habla la lengua extranjera mediante la presentación de temas de actualidad y actividades lúdicas.

6. Llevar a cabo cualquier otra actividad que le sea encomendada por la Consejería de Educación de la Junta de Andalucía.

Teniendo en cuenta todo lo anterior, un auxiliar, como el resto de los profesores y profesoras, además debe tener en cuenta las necesidades, intereses, habilidades y capacidades de los alumnos.

Esto se refiere tanto a la lengua como a los contenidos o temáticas y a la metodología. La clase debe desarrollarse en un clima cálido, que brinde seguridad y confianza al alumnado. Para 
ello, el aula debe contar de un ambiente atractivo y acogedor que favorezca el acercamiento a la cultura del país cuya lengua se estudia con posters, fotografías, mapas... que puede aportar el mismo auxiliar o trabajos de los niños y niñas.

El auxiliar no debe olvidar en ningún momento que debe facilitar gran cantidad de input comprensible para así alimentar los mecanismos inconscientes con suficientes ejemplos de la lengua y hacer que los alumnos los comprendan y debe tratar los errores como necesarios en el proceso que implica el aprendizaje de una lengua.

\section{4.- BENEFICIOS DE TENER UN AUXILIAR DE CONVERSACIÓN EN EL AULA.}

Un auxiliar de conversación es una persona cuyo perfil puede ser muy variado, es decir, no tiene porque responder a un patrón en concreto. Generalmente se trata de jóvenes de entre 18 y 30 años, cuya titulación universitaria puede ser cualquiera y su país de destino también, siempre y cuando, su lengua materna corresponda con el idioma extranjero que se enseña en el centro y al mismo tiempo tenga un mínimo de conocimientos sobre la lengua del país con el que va a colaborar.

Procedentes la mayoría de países como Gran Bretaña, Irlanda, Estados Unidos, Canadá, Francia, Italia o Alemania, los auxiliares de conversación llevan ya colaborando en nuestro país alrededor de una década con los profesores de idiomas en los centros educativos, para mejorar las competencias idiomáticas del alumnado a la vez que ellos mejoran también al mismo tiempo, su español y aumentan sus conocimientos sobre la cultura española y la comunidad autónoma a la que han sido destinados.

\begin{tabular}{llcc} 
Este tipo de de & \multicolumn{3}{c}{ Tabla 1.- Sistema en Reino Unido. } \\
\cline { 2 - 4 } "profesorado" como se & & Edad (Age) & Cursos (Years) \\
\cline { 2 - 4 } les podría calificar, por & KS1 & $5-7$ & 1 y 2 \\
regla general, dominan & KS2 & $7-11$ & $3,4,5$ y 6 \\
el español aunque & KS3 & $11-14$ & 7,8 y 9 \\
apenas hacen uso de él & KS4 & $14-16$ & 10 y 11 \\
durante las lecciones y & KS
\end{tabular}

Gracias a ellos, en la mayoría de las ocasiones, los alumnos y alumnas encuentran el grado de motivación que les falta a la hora de aprender un segundo idioma ya que tienen la posibilidad de tener contacto y una exposición directa con la lengua extranjera a través de ellos y transmiten al resto de la comunidad, la necesidad de aprender lenguas, ofreciendo contextos reales en los que la situación comunicativa no es una mera simulación, obligando al otro interlocutor, ya sean los propios alumnos o alumnas o incluso el profesor o profesora, a realizar un esfuerzo por expresarse y comprender.

Asimismo, los alumnos y alumnas pueden perfeccionar no sólo los aspectos gramaticales de la lengua, sino también los fonéticos, pronunciación, a la vez que conocen la realidad cultural del país de origen del auxiliar de conversación en cuestión.

\section{5.- MI PROPIA EXPERENCA COMO AUXILIAR DE CONVERSACIÓN.}

Durante el curso académico 2007/2008, el programa de Auxiliares de Conversación del MEC me brindó la oportunidad, bien aprovechada, de vivir un año diferente en el extranjero con el objetivo de enseñar lengua y cultura española en una ciudad del Reino Unido. Tras ser seleccionada y admitida para una de las 356 plazas que se ofertaban aquel año para países de lengua inglesa, mi destino fue la ciudad de Crawley, al sur de Inglaterra.

No tuve demasiadas dificultades en adaptarme al nuevo país ya que lo conocía de años anteriores y la bienvenida que me brindaron en los colegios donde trabaja fue maravillosa. Sin embargo, en lo que sí me sentí un poco desubicada al principio fue en lo relativo al funcionamiento de los colegios de allí. Y es que en el sistema educativo británico, se puede apreciar alguna que otra diferencia en comparación con el español.

En primer lugar, en lo que a la escolarización de los niños y niñas se refiere, se utiliza como referente el año académico y no el natural. Así, los niños nacidos en julio pertenecerían a un curso anterior a los nacidos en agosto. 
En cuanto al sistema (tabla 1), se divide en Key Stages y Years, que se corresponderían con nuestros ciclos y cursos, respectivamente. En el Reino Unido hay cuatro Key Stages, lo que en términos comparativos equivaldría a tener un ciclo más que nosotros, y once Years, que se distribuyen del siguiente modo:

Las asignaturas se dividen en Core Subjects (equivalentes a lo que entenderíamos por asignaturas fundamentales o troncales para todos los ciclos) y Foundation Subjects (se trata de asignaturas que no se encuentran en todos los ciclos). Además, encontramos la asignatura P.S.H.E (Educación personal, social y para la salud), que se considera transversal, y Educación Religiosa, que trata del estudio de otras religiones y tiene carácter optativo.

Las Core Subjects son Inglés, Matemáticas y Ciencias; las Foundation Subjects son Diseño y Tecnología, Tecnología de la Comunicación y la Información (que se considera cada vez más como Core Subject), Historia, Geografía, Lengua extranjera, Arte y Diseño, Música, Educación Física y Educación cívica. En cuanto a esta distribución cabe destacar algunos aspectos que resultan llamativos en comparación con nuestro sistema:

- En el último ciclo (KS 4, equivalente a nuestro $3^{\circ}$ y $4^{\circ}$ de ESO) no se da Geografía ni Historia.

- La lengua extranjera comienza a impartirse en el KS1 o incluso antes.

- Se oferta la educación sexual como asignatura optativa.

En lo referente a la planificación de la asignatura, allí se planifica mucho. Todo el profesorado tiene que hacerlo para cada clase, pero supervisado por la dirección del centro, pues antes del inicio de la semana deben haber enviado su planificación semanal al director.

Al igual que en España, allí también existe una Planificación Anual, pero la diferencia radica en que en medio de cada trimestre hay una semana de vacaciones utilizada para planificar más específicamente. Son programaciones de medio trimestre y hay seis en total.

Otra de las cosas más llamativas y características que se puede observar en cualquier colegio británico concierne a los objetivos de aprendizaje, y es que al principio de cada clase, el profesor o profesora escribe en la pizarra los objetivos específicos que el alumnado debe alcanzar al finalizar cada clase con objeto de que los tengan claros y presentes.

Por último, y a pesar de que aún se podrían describir muchas más diferencias, no me gustaría dejar sin mencionar la importancia de una serie $d$ exámenes que se realizan en determinadas etapas en cada colegio. Se trata de los SATs, GCSE y "A" Levels exámenes, que tienen como finalidad evaluar el nivel de los alumnos al finalizar una etapa educativa y requisito indispensable para continuar estudiando dentro del sistema reglado.

Mi labor como profesora se repartía entre dos colegios diferentes, el Oriel High School (colegio de Educación Secundaria) y Maidenbower Junior School (colegio de Educación primaria). Allí, mi tarea era principalmente trabajar la comunicación oral con los alumnos.

Trabajaba doce horas a la semana, tal y como se estipula en el contrato de cualquier auxiliar, aunque en muchas ocasiones me quedaba al terminar ayudando al profesor $\mathrm{o}$ profesora que tocara a preparar actividades para la clase del día siguiente o aportando ideas para futuras actividades.

Desde el primer día se me pidió que aportara siempre que fuera posible recursos made in Spain, es decir, traídos directamente de España, por lo que cada vez que tenía la oportunidad de viajar a mi ciudad, volvía cargada con pósters de ciudades, folletos publicitarios, postales típicas, cárteles de películas u obras de teatro, menús de restaurantes.... Que a todos los alumnos y alumnas le causaban mucho Itnez y que utilizábamos además para decorar las aulas.

El profesorado estaba muy preparado en cuanto a los conocimientos teóricos en español, gramáticas, fonética... sin embargo las clases orales dejaban mucho que desear.

En mis clases el diálogo primaba por encima de todo. A través de talleres fundamentalmente prácticos, los alumnos aprendían a utilizar la TV, Internet, el cine, la publicidad, la prensa y la música en la lengua real y no sólo en la académica del aula.

Casi siempre trabajábamos sobre temas de actualidad en el mundo, sobre todo en España, y comentábamos las distintas tradiciones y fiestas que tenemos en nuestro país, cantábamos canciones, decíamos refranes, imposibles trabalenguas y hasta chistes. 
El tema de las vacaciones era uno de los más frecuentes entre el alumnado y era muy curioso el hecho de que más del $90 \%$ de ellos había viajado a España en alguna ocasión y alrededor del 50\% tenía un familiar viviendo en nuestro país o una residencia de verano aquí.

La ayuda del resto del profesorado del departamento de lenguas modernas y la buena disposición de los alumnos y alumnas, hacía que cada día el trabajo fuera más satisfactorio y agradable cada día.

Aquel año, bajo mi punto de vista, todo el alumnado aprendió mucho, ya que es un privilegio poder practicar un idioma cada día con una persona que es nativa del país de origen de dicho idioma.

\section{6.- CONCLUSIONES.}

Gracias a todas las medidas mencionadas en este artículo y que se están llevando a cabo desde el Gobierno para fomentar el aprendizaje de idiomas en los centros de enseñanza españoles, se está haciendo un verdadero esfuerzo para poner a los alumnos al mismo nivel idiomático que el resto de los europeos y compensar así, las tres décadas de retraso que lleva el país en este sentido.

La figura de un auxiliar de conversación en el aula aporta muchos beneficios tanto para los alumnos como para los profesores, dando una mayor calidad a la competencia lingüística que de otra forma sería difícil de conseguir. De alguna manera y gracias también al apoyo de estos colaboradores lingüísticos, se fomenta el acceso a la ya mencionada interculturalidad cada vez más presente en los colegios y se inculca al alumnado un conocimiento diferente sobre otra cultura que va más allá de los propios libros de texto.

Estudiar una lengua extranjera significa adquirir ciertas competencias de comunicación básicas, estas son, leer, escribir, hablar, comprender.... Pero también es muchos más que eso, se trata de acercarse a la cultura del otro, adquirir otra forma de ver el mundo, entrar en contacto con textos, autores, otras maneras de pensar... Y por esto, el hecho de tener a una persona nativa en las clases de idiomas, aporta una serie de beneficios, que con la figura sola del profesor en el aula, serían más difíciles de conseguir.

Sin embargo, a parte de todos los planes que se están llevando a cabo para que los jóvenes españoles sean más competentes en cuanto idiomas, no se debería dejar en el olvido a los docentes. Y no es que no estén lo suficientemente bien preparados para enseñar un idioma, que lo están y cada vez es más obvio, sino que para todos aquellos que nacieron unas cuantas décadas más atrás o para los que no están muy familiarizados con las nuevas innovaciones en la materia, deberían recibir algún tipo de formación intercultural para poder afrontar de una manera más completa una lección en un idioma extranjero, así como ofrecer a los alumnos y alumnas propuestas que hagan la clase más apetecible.

\section{7.- REFERENCIAS BIBLIOGRÁFICAS.}

Byran, M. \& Fleming M, (1998). Perspectivas interculturales en el aprendizaje de idiomas. Cuadernos Cervantes de la lengua española. Press Syndicate of the University of Cambridge.

Cobo, F., Motos, M. A., García A. \& Moral, G. (2009), Análisis de las actividades realizadas en los proyectos de interculturalidad desarrollados en las provincias de Almería y Málaga. CuevasOlula (Almería): Revista Espiral, Cuadernos del profesorado, 2 (3), 104-110.

Comisión Europea (2006). Los europeos y sus lenguas: Encuesta Eurobarómetro especial 243. [en línea]. Disponible en: http://ec.europa.eu/public opinion/archives/ebs/e bs_243_sum_es.pdf [Consulta 2009, 2 de diciembre].

Consejería de Educación de la Junta de Andalucía (2008). Plan de Fomento del Plurilingüismo.[en línea]. Disponible en http://www.juntadeandalucia.es/compromisos200 82012/archivos_repos/0/140.pdf. [Consulta 2009, 6 de diciembre].

IV Congreso Internacional de Educación Intercultural (noviembre, 2009). Nuevos retos internacionales ante la interculturalidad [en línea]. Disponible en:

http://www.ual.es/GruposInv/EducacionIntercult ural/ [Consulta 2009, 16 de diciembre].

Orden del 14 de septiembre de 2007 por la que se modifica la del 20 de junio de 2006, por la que se regula la provisión y actividad de los auxiliares de conversación en los centros docentes públicos de la Comunidad Autónoma de Andalucía. Boletín Oficial de la Junta de Andalucía, de octubre de 2007.

Orden del 20 de junio de 2006 por la que se regula la provisión y actividades de los auxiliares de conversación de los centros públicos de la Comunidad Autónoma de Andalucía- Boletín Oficial de la Junta de Andalucía de 10 de julio de 2006. 
Ramos, F. (2007). Programas bilingües y formación de profesores en Andalucía. Revista Ibero Americana de Educación [en línea] 44. Disponible en: http://www.rieoei.org/rie44a07.htm [Consulta 2009, 10 de diciembre].
Swarts, S. (2009). What is a language assistant? Suite 101 [en línea]. Disponible en: http://teachingabroad.suite101.com/article.cfm/what is a langu age_assistant [Consulta: 2009, 6 de diciembre].

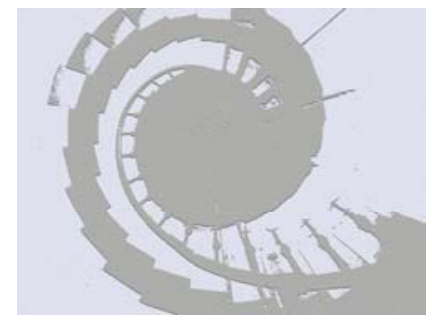

